Ann. Zootech., I97I, 20 (3), 29I-304.

\title{
INFLUENCE DU POLOXALÈNE SUR LES MÉTÉORISATIONS, LES QUANTITÉS D'ALIMENT INGÉRÉES ET LA CROISSANCE DE TAURILLONS RECEVANT DES RATIONS CONDENSÉES
}

\author{
J. FREBling, P. LARVOR *, C. MALTERRE **, M. AZAN ***,
} J. GAILLARD *** et M. HENNEQUIN ****

avec la collaboration technique de A. Clerlsoli et Y. Guérin

Station de Génétique quantitative et appliquée,

Centre national de Recherches zootechniques, I. N.R. A., 78 - Jouy-en-Josas

* Station de Physiopathologie de la Nutrition,

** Station de Recherches sur l'Élevage des Ruminants, Centre de Recherches de Clermont-Ferrand, I. N.R. A., 63 - Saint-Genès-Champanelle

*** Institut technique de l'Élevage bovin, 41, rue de la Bienfaisance, 75-Paris (8e)

**** Coopérative d'Élevage du Sud-Onest, Domaine de Tournal, 81 - Soual

\section{RÉSUMÉ}

Au cours de deux expériences successives nous avons étudié l'influence du poloxalène sur les météorisations, les quantités d'aliments ingérées et la croissance de I 23 taurillons recevant des rations condensées.

L'incorporation de poloxalène dans la ration (utilisé à la dose de o, I et o,2 p. Ioo) entraîne sur l'ensemble de la durée expérimentale :

- une diminution significative du nombre de cas de météorisations et en particulier du nombre de cas graves dont la fréquence augmente notablement dans les lots témoins lorsque le régime se prolonge au-delà de 3 mois environ,

- une augmentation significative de la quantité moyenne d'aliment ingérée,

- une augmentation significative du gain de poids moyen quotidien, liée à l'accroissement de la consommation.

L'utilisation de telles substances, qui diminuent l'accumulation des gaz dans le rumen, permet d'envisager une nouvelle méthode pour accroître l'appétit des ruminants. 


\section{INTRODUC'TION}

L'alimentation des ruminants avec des rations condensées (broyées et agglomérées), contenant 30 à 80 p. roo de céréales, permet l'obtention d'une croissance très rapide. Toutefois, elle entraîne fréquemment des troubles variés, dont la description a fait l'objet de quelques revues récentes (LARVor, I967; FERrando, I968). Parmi ces perturbations, la météorisation, chronique ou aiguë, est l'une des plus graves.

La météorisation résulte de 1'accumulation dans le rumen de gaz non évacués. Sa cause peut donc être, soit un obstacle à l'évacuation des gaz, soit une production excessive de ces gaz, les deux phénomènes pouvant être concomitants. Le plus souvent la météorisation est liée à l'ingestion massive de légumineuses à certains stades de végétation (parfois aussi de jeunes graminées). Ces plantes étant constituées principalement de substances rapidement fermentescibles, l'attaque microbienne est rapide, avec un dégagement de gaz important. Mais le facteur primordial est le blocage de l'éructation qui peut éventuellement résulter de la présence de substances toxiques dans le fourrage, mais est généralement dû à un phénomène nerveux : le réflexe d'éructation est bloqué lorsque le niveau du cardia est dépassé par le contenu du rumen. Dans des conditions normales ce remplissage excessif ne peut pas se produire, mais si la tension superficielle et la viscosité du contenu du rumen sont anormales, les gaz de fermentation provoqueront alors la formation d'une mousse stable qui va remplir la partie libre du rumen et inhiber l'éructation.

Le risque de météorisation dépend donc de la tension superficielle du contenu du rumen qui résulte d'un équilibre entre la présence dans l'aliment de quantités plus ou moins grandes de substances moussantes (protéines solubles, pectines, saponine) et de l'arrivée dans le rumen d'un anti-moussant naturel très puissant, les mucines salivaires (BARTLEY et YADAVA, I96I ; VAN HORN et BARTLEY, I96I).

Cependant, la sécrétion salivaire est diminuée lors de la consommation d'aliments riches en eau tels que les jeunes légumineuses et la quantité de salive peut devenir insuffisante pour empêcher la formation de mousses. Ainsi, la production de salive par $\mathrm{kg}$ de matière sèche ingérée de luzerne à maturité (stade non précisé par l'auteur) est de $3,3 \mathrm{~kg}$ contre $2,7 \mathrm{~kg}$ pour la luzerne jeune (MEYER et al., I964). De plus, la comparaison d'animaux très sensibles à la météorisation avec des animaux peu sensibles a montré que ces derniers salivaient plus abondamment (MENDEL et BODA, I96I).

Dès lors, les travaux s'orientèrent vers la recherche d'un produit capable de simuler les effets tensio-actifs de la salive, de synthèse facile, ayant un goût accepté par les animaux et non résorbé par le tube digestif des ruminants.

Le poloxalène, copolymère de polyoxyéthylène et polyoxypropylène, substance tensio-active non ionique de haut poids moléculaire, présente semble-t-il ces caractéristiques. Il s'est révélé être un agent de prévention efficace de la météorisation par les légumineuses (BARTLEY, I965 $a$ et $b$; BARTLEY et al., I965) ainsi qu'un agent thérapeutique valable (BARTLEY et al., I967). Le mécanisme de son action dépend bien de sa capacité à modifier la tension superficielle, et ne doit rien à une quelconque 
modification des fermentations du rumen : il est purement physique (HELMER et al., I965). Sa résorption et son passage dans le lait et les tissus mesurés au moyen de poloxalène ${ }^{14} \mathrm{C}$, sont négligeables (Mryer et al., I965).

La météorisation observée après distribution d'une ration condensée ne semble pas très différente en nature de celle due aux légumineuses. De tels aliments se délitent rapidement dans le rumen offrant aux bactéries une surface d'attaque considérable et souvent une forte proportion de nutriments rapidement fermentescibles. Ainsi avec la luzerne broyée il en résulte une augmentation de la production de gaz, mise en évidence par incubation in vitro du contenu du rumen (JOURNET et DEMARQUILI,Y, I967), d'autant plus importante que les particules sont plus fines et la proportion de céréales plus élevée (MaLterrre et al., I969). D’un autre côté, la réduction du temps de rumination avec de telles rations (KICK et GERLAUGH, I936; Gordon, I958; DEMARQUili,y et JoURNET, I967; RUCKEBusch, I967) a pour conséquence une réduction de la sécrétion salivaire (PuTnam et al., I966). On retrouve donc le même phénomène de tension superficielle anormale du contenu du rumen, peut-être aggravé par la diminution de la motricité des préestomacs et la réduction du temps de rumination (RUCKebUSCH, I967). Le poloxalène s'est également révélé efficace dans la prévention et le traitement de la météorisation par ces rations riches en céréales (Shone, I965; Geissler et Thomas, ig66), bien que l'effet soit peut-être un peu moins net que dans la météorisation due aux légumineuses en vert (BARTLEY et MEYER, Ig68).

Compte tenu de ces résultats, nous avons essayé de mesurer l'influence du poloxalène sur les météorisations de taurillons recevant des régimes condensés. Ces animaux, futurs reproducteurs, soumis au contrôle de performances, reçoivent une ration condensée standard qui permet d'extérioriser au maximum leur potentiel de croissance mais en entraînant parfois des météorisations.

\section{MATÉRIEL ET MÉTHODES}

Pour mettre en évidence l'influence du poloxalène sur l'apparition des météorisations, nous avons constitué des lots de taurillons âgés de ro mois environ comparables selon leur race, leur poids et leur âge : les animaux ont été répartis en deux lots, l'un recevant du poloxalène, l'autre n'en recevant pas ct constituant ainsi le lot témoin.

Nous avons réalisé deux expériences au cours de deux années successives ; les effectifs, les caractéristiques des animaux et les durées de contrôle sont reportés sur le tableau $\mathbf{I}$.

$\mathrm{Au}$ cours de la première expérience nous avons utilisé 51 taurillons de race Charolaise; dans la seconde, les animaux appartenaient à deux types génétiques : $3^{8}$ Aubrac $\times$ Garonnais et 38 A ubrac $\times$ Charolais répartis également selon les deux lots.

Alimentation et conditions de vie

Les animaux ont tous été conduits dans les mêmes conditions pour une même expérience.

Dans la première, les taurillons Charolais étaient en stabulation entravée, sur litière de paille et recevaient à volonté ( 5 à Io p. Ioo de refus), en deux distributions, un aliment condensé broyé et aggloméré dont la composition était la suivante :

- 67 p. Ioo de farine de luzerne déshydratée,

- 30 p. roo d'orge,

3 p. Ioo de complément minéral vitaminisé comprenant : $5^{\circ} \mathrm{p}$. Ioo phosphate disodique, 45 p. Ioo chlorure de sodium, 4 p. I oo sulfate de fer, I p. Ioo sulfate de manganèse $\left(3 \mathrm{H}_{2} \mathrm{O}\right)$, o, I p. I oo sulfate de cuivre $\left(5 \mathrm{H}_{2} \mathrm{O}\right)$, I80 ooo UI vitamine $A$ et 20 ooo UI vitamine $\mathrm{D}_{2}$ par $\mathrm{kg}$ de complément minéral. 
Le poloxalène, présenté en poudre contenant environ $5^{\circ} \mathrm{p}$. Ioo de produit pur, était incorporé à raison de 2 grammes par $\mathbf{k g}$ d'aliment.

TABLEAU I

Caractéristiques des expériences

\begin{tabular}{|c|c|c|c|c|}
\hline Caractéristiques & \multicolumn{2}{|c|}{$1^{\mathrm{re}}$ expérience } & \multicolumn{2}{|c|}{2 expérience } \\
\hline $\begin{array}{l}\text { Annce } \\
\text { Lieu }\end{array}$ & \multicolumn{2}{|c|}{$\begin{array}{c}\text { Hiver } 1967-1968 \\
\text { Osmoy (Cher) }\end{array}$} & \multicolumn{2}{|c|}{$\begin{array}{l}\text { Hiver } 1968-1969 \\
\text { Soual (Tarn) }\end{array}$} \\
\hline Lots & Témoin & "Poloxalène " & Témoin & "Poloxalène" \\
\hline $\begin{array}{l}\text { Nombre d'animaux mis en expé- } \\
\text { rience } \ldots \ldots \ldots \ldots \ldots \ldots \ldots \ldots \ldots \ldots \ldots \\
\text { Nombre d'animaux éliminés en } \\
\text { cours d'expérience } \ldots \ldots \ldots \ldots \ldots\end{array}$ & $\begin{array}{r}26 \\
1\end{array}$ & 25 & $\begin{array}{l}38 \\
1\end{array}$ & $\begin{array}{l}38 \\
0\end{array}$ \\
\hline Race $\ldots \ldots \ldots \ldots \ldots \ldots \ldots \ldots$ & Charolais & Charolais & $\begin{array}{c}\text { Aubrac } \times \\
\text { Garonnais } \\
\text { Aubrac } \times \\
\text { Charolais }\end{array}$ & $\begin{array}{l}\text { Aubrac } \times \\
\text { Garonnais } \\
\text { Aubrac } \times \\
\text { Charolais }\end{array}$ \\
\hline 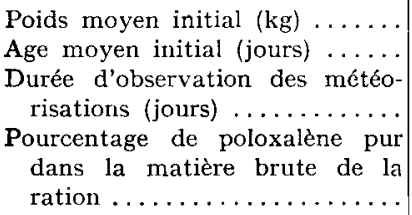 & $\begin{array}{c}425 \pm 51 \\
318 \pm 23 \\
78(25 \text { animaux }) \\
126(16 \text { animaux })\end{array}$ & $\begin{array}{c}423 \pm 60 \\
31 \pm \pm 25 \\
78(21 \text { animaux }) \\
126(14 \text { animaux })\end{array}$ & $\begin{array}{c}317 \pm 38 \\
307 \pm 17 \\
126\end{array}$ & $\begin{array}{c}315 \pm 29 \\
306 \pm 15 \\
126\end{array}$ \\
\hline
\end{tabular}

Dans la deuxième expérience, les animaux étaient menés en lots de 4 ou 5 sur litière de paille, en stabulation libre; ils recevaient également à volonté ( 5 à io p. roo de refus) au cours de deux repas, un aliment condensé de la composition suivante :

- $64 \mathrm{p}$. roo de foin de luzerne $\mathrm{I}^{\mathrm{re}}$ coupe,

- 30 p. I00 de maïs,

- 2 p. Ioo de mélasse,

- 4 p. Ioo de complément minéral vitaminisé comprenant : $55 \mathrm{p}$. Ioo phosphate bicalcique, $4^{\circ} \mathrm{p}$. Ioo chlorure de sodium, $5 \mathrm{p}$. Ioo sulfate de fer, o, I p. Ioo sulfate de cuivre $\left(5 \mathrm{H}_{2} \mathrm{O}\right), 0,4 \mathrm{p}$. IOo sulfate de manganèse $\left(3 \mathrm{H}_{2} \mathrm{O}\right)$, I 80 ooo UI vitamine $\mathrm{A}, 4$ o ooo UI vitamine $\mathrm{D}_{2}$.

Le poloxalène sous forme huileuse et pure était incorporé à raison de $2 \mathrm{~g}$ de produit pur par kg d'aliment.

Entre leur arrivée et le début de l'expérience, les animaux sont restés en stabulation libre et ont reçu du foin et l'aliment condensé selon des quantités respectivement décroissantes et croissantes; cette période a duré de 30 à 40 jours.

\section{Météorisations.}

Mesures

Un observateur unique pour chaque expérience notait chaque jour et pour chaque animal l'absence ou la présence de météorisations ainsi que l'intensité de ces dernières selon le barême ci-dessous :

o : absence de météorisation,

I : léger ballonnement unilatéral,

2 : fort ballonnement unilatéral,

3 : léger ballonnement bilatéral,

4 : fort ballonnement bilatéral,

5: météorisations nécessitant une intervention (sondage ou trocard). 
La durée d'observation a été de 126 jours sauf dans la première expérience pour ıo animaux du lot témoin et ro du lot traité contrôlés seulement pendant les 78 derniers jours.

\section{Croissance.}

Les animaux ont été pesés trois jours consécutifs au début et à la fin de chaque période expérimentale et tous les I 4 jours dans l'intervalle des 126 jours.

Ces pesées ont été utilisées pour calculer le gain de poids vif des animaux et établir leur courbe de croissance.

\section{Quantités ingévées.}

Les quantités d'aliment ingérées ont été mesurées tous les jours, soit individuellement au cours de la première expérience soit par lots au cours de la seconde. Ces mesures ont été effectuées sur la base de la matière brute, la teneur en matière sèche de l'aliment étant d'environ 88 p. Ioo.

Abattage.

Nous avons étudié seulement les carcasses des taurillons de la deuxième expérience selon le protocole retenu pour les animaux du contrôle de descendance (FreBLing et al., r967).

\section{RÉSULTATS}

\section{Météorisations}

Dans la première expérience I animal du lot témoin et 2 animaux du lot recevant le poloxalène ont dû être éliminés en cours de contrôle en raison des risques de météorisations mortelles (cf. tabl. I). Dans la seconde, un animal du lot témoin est mort à la suite de météorisations (1).

Pour comparer les deux lots nous avons, dans une première analyse, calculé une note moyenne $\mathrm{X}$ par animal pour la totalité de la période d'observation, tenant compte à la fois de la fréquence et de l'intensité des météorisations tel que :

$$
\begin{gathered}
i=5 \\
\mathrm{X}=\frac{i n_{i} \cdot i}{\mathrm{~N}} \mathrm{I}^{-}
\end{gathered}
$$

$i=$ intensité des météorisations notée de $\mathrm{I}$ à 5

$n_{i}=$ nombre de cas de météorisations d'intensité $i$

$\mathrm{N}=$ nombre de jours d'observations.

Les notes moyennes pour les animaux des lots témoins se sont élevées respectivement à 0,23 et 0,63 pour les première et seconde expériences contre 0,16 et 0,52 pour les animaux des lots traités.

Nous avons ensuite regroupé les notes moyennes en classes, selon le tableau 2.

(1) Les observations des météorisations sur ces animaux figurent dans les résultats jusqu'à leur disparition du contrôle tandis qu'ils n'ont pas été inclus dans les résultats concernant la croissance et les quantités ingérées. 
Il n'est pas apparu, sur ces répartitions en classes, de différences significatives (test du $\chi^{2}$ ) entre les lots témoin et traité, tant dans la première que dans la seconde expérience.

TABI,EAU 2

Nombre d'animaux par classe de notes

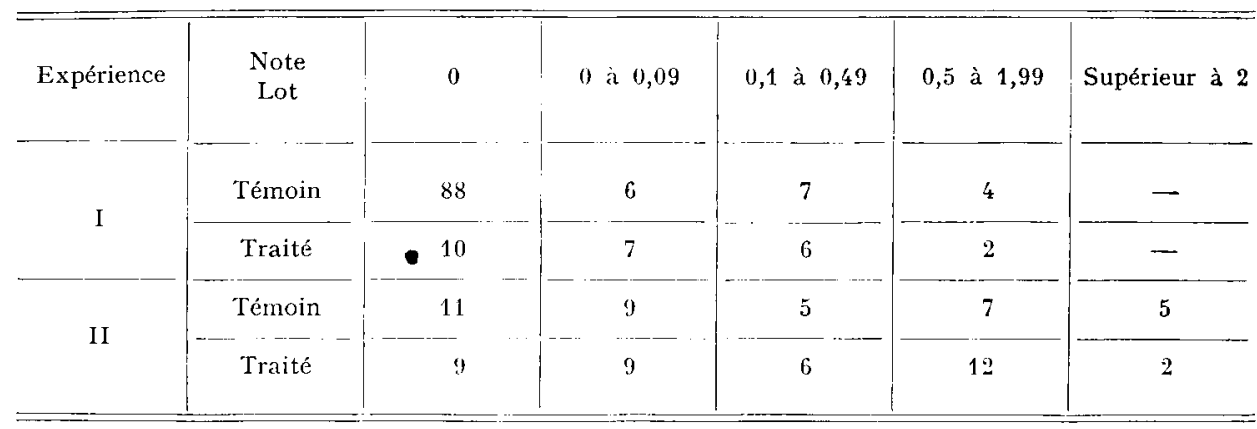

Dans une seconde analyse nous avons considéré séparément les météorisations d'intensités I, 2, 3-4-5 regroupées. Nous avons calculé pour chacune de ces classes et pour la totalité des animaux d'un même lot la fréquence des météorisations, c'està-dire le nombre de cas de météorisations rapporté au nombre total d'observations (nombre de jours d'observation $\times$ nombre d'animaux observés). Les résultats de cette analyse figurent sur le tableau 3 .

\section{TABLEAU 3}

Fréquence des météorisations observées en fonction de leur intensité

(Nombre de cas de météorisations en p. Ioo du nombre total d'observations)

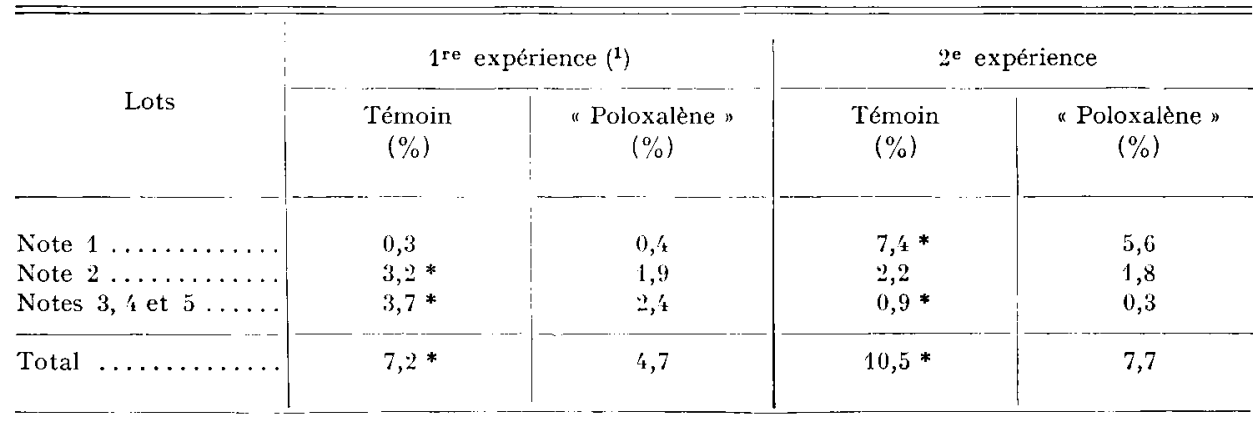

(1) Au cours de la première expérience, la période d'arrêt de distribution du poloxalène est exclue des résultats.

* Différence significative au seuil de 5 p. 100 (calculs effectués par la méthode du test de $x^{2}$ réalisé en considérant les fréquences des météorisations observées du lot témoin comme fréquence théorique).

En outre, pour décrire l'évolution de ces météorisations, nous avons calculé et représenté graphiquement le nombre de cas de météorisations observés par semaine (nombre d'animaux qui ont météorisé $\times$ nombre de jours oì l'on a observé ces météorisations sur chacun d'eux). 

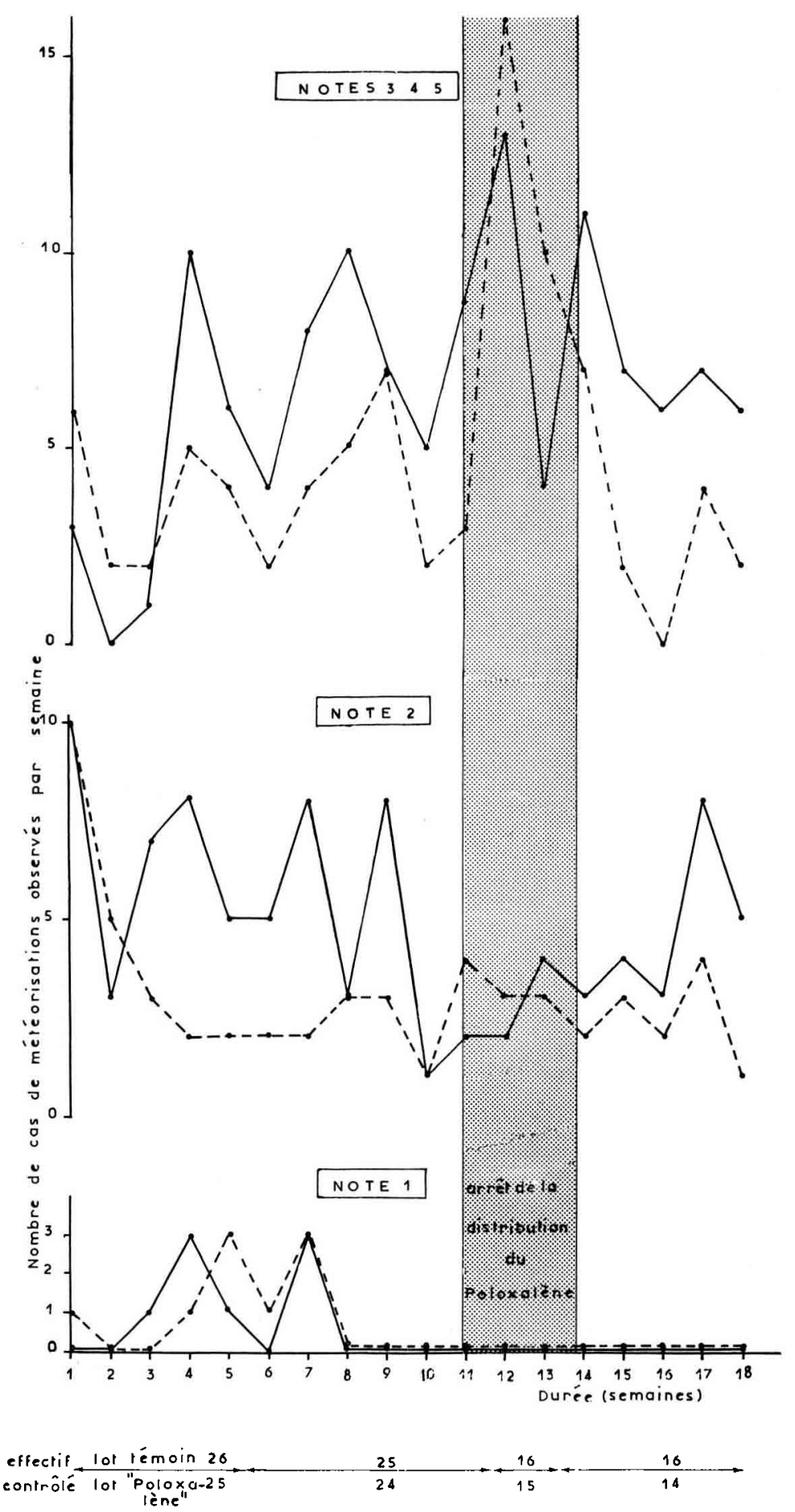

FIG. I. - Évolution des météorisations selon leur intensité (I re expérience) - Lot témoin
$-\ldots$ Lot "Poloxalène " 

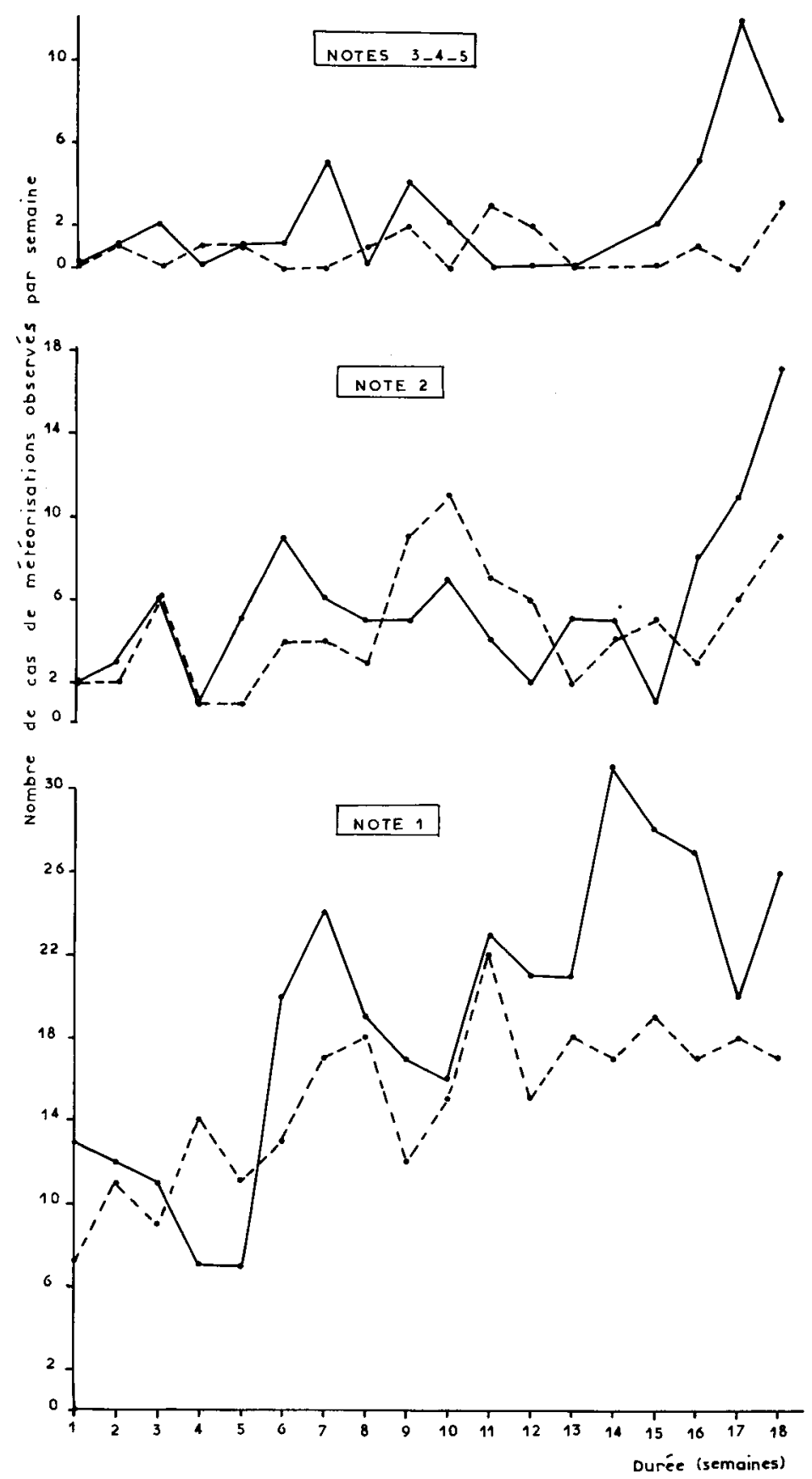

effectif

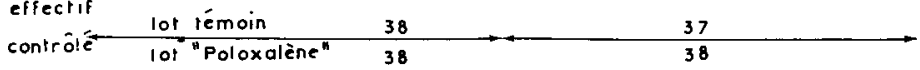

FIG. 2. - Evvolution des météorisations selon leur intensité ( $2^{\mathrm{e}}$ expérience) _- Lot témoin 
Dans ces analyses nous n'avons pas distingué la part respective du nombre d'animaux qui météorisent et du nombre de cas de météorisation par animal.

\section{Première expérience.}

Les résultats obtenus au cours de cette première expérience (tabl. 3, fig. I) permettent de mettre en évidence un effet significatif sur la fréquence totale des météorisations durant les $I 5$ semaines où les animaux ont reçu le poloxalène $(4,7 \mathrm{p}$. Ioo contre 7,2 p. Ioo pour le lot témoin). Cette réduction est significative tant dans les cas d'intensité 2 que dans les cas d'intensité 3 et au-dessus. Mais cela résulte d'un effet très notable sur seulement quelques semaines.

Pendant la période d'arrêt de distribution du poloxalène aucune différence significative n'existe entre les deux lots, le nombre de cas, particulièrement le nombre de cas graves étant cependant supérieur pour le lot antérieurement complémenté au poloxalène qui semblerait donc ne pas avoir d'effet rémanent.

\section{Deuxième expérience.}

Dans la seconde expérience (tabl. 3, fig. 2), la distribution de poloxalène a permis également de réduire de façon significative la fréquence totale des météorisations durant les I 8 semaines d'observations ( 7,7 p. roo contre I0,5 p. Ioo pour le lot témoin). Cette réduction est significative pour les cas d'intensité I et 3-4-5 et se manifeste particulièrement durant les 5 dernières semaines d'observation.

Il semble donc se dégager de ces deux expériences un effet significatif du poloxalène sur l'apparition de météorisations au cours de l'ensemble de la période expérimentale. Cet effet est particulièrement important pour les cas graves dont la fréquence augmente notablement en fin de période dans les lots témoins.

Enfin, il est important de signaler qu'aucune comparaison ne peut être faite sur les fréquences des météorisations entre les deux expériences ; en effet, l'observateur

à̀re expérience

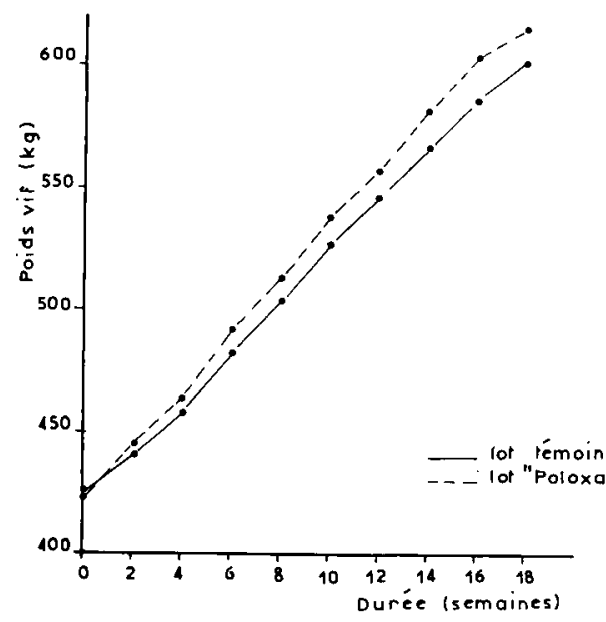

Fig. 3 .
2 ème expérience

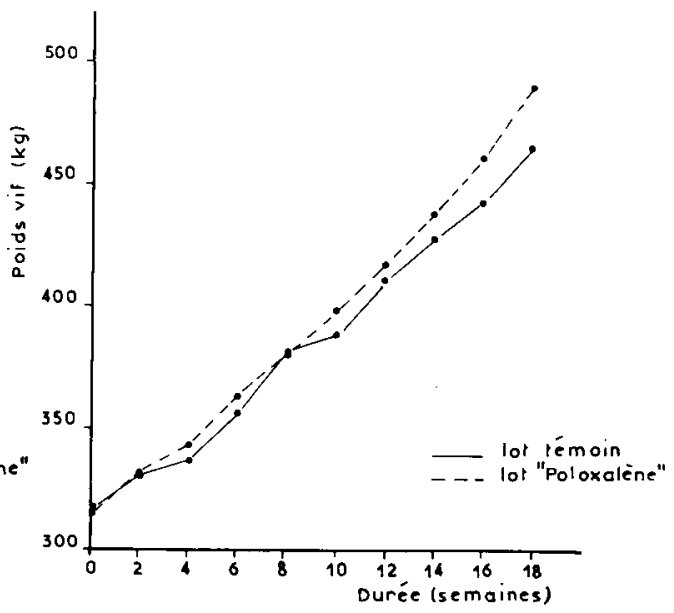

Courbes de croissance 
de $1 \mathrm{a} 2^{\mathrm{e}}$ expérience a été plus sévère dans ses notations que celui de la $\mathrm{I}^{\mathrm{re}}$, les taux d'incorporation du poloxalène ont été différents ainsi que le type, le poids, 1'âge et l'origine des animaux utilisés.

\section{TABLEAU 4}

Gain de poids et quantités ingérées (126 jours)

\begin{tabular}{|c|c|c|c|c|}
\hline \multirow{2}{*}{ Lots } & \multicolumn{2}{|c|}{$1^{\text {re }}$ expérience } & \multicolumn{2}{|c|}{$2^{\text {e }}$ expérience } \\
\hline & Témoin & "Poloxalène " & Témoin & "Poloxalène" \\
\hline Nombre d'animaux & 25 & 23 & 37 & 38 \\
\hline $\begin{array}{l}\text { Gain moyen quotidien }\left({ }^{1}\right) \\
(\mathrm{g} / \mathrm{j})\end{array}$ & $1408 \pm_{-} 183$ & $1536 \pm 200^{*}$ & $1178 \pm 236$ & $1370 \pm 155^{* *}$ \\
\hline $\begin{array}{c}\text { Quantités ingérées } \\
\text { (kg de matière brute/j) }\end{array}$ & $10,00 \pm 1,31$ & $10,98 \pm 1,37 *$ & $10, \frac{1}{4} 0$ & $11,45 *$ \\
\hline $\begin{array}{c}\text { Efficacité alimentaire } \\
\text { (kg d'aliment/kg de gain) }\end{array}$ & $7,17 \pm 1,03$ & $7,21 \pm 0,90$ & 8,81 & 8,36 \\
\hline
\end{tabular}

(1) La comparaison des gains moyens quotidiens entre les 2 lots a été réalisée. Dans les deux expériences, par analyse de variance en décomposant la variance totale en variance entre lots (témoin et "poloxalène ") et variance à l'intérieur des lots.

* Différence significative au seuil de 5 p. 100.

** Différence significative au seuil de 1 p. 100.

Tère expérience

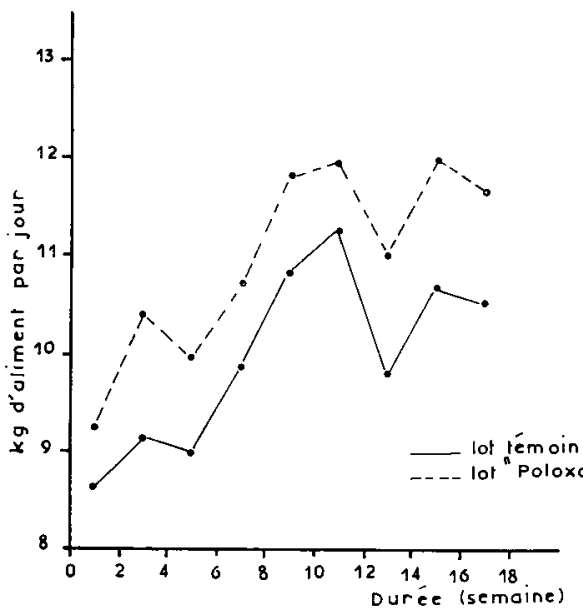

zème expérience

FIG. 4. - Evolution des quantités ingérées 


\section{Croissance}

Les résultats obtenus au cours des deux expériences concordent (tabl. 4, fig. 3). Les animaux des lots recevant le poloxalène ont eu un gain moyen quotidien significativement supérieur à celui des animaux des lots témoins : + 9, I p. Ioo dans la première expérience $(P<0,05)$ et de $+16,3$ p. Ioo dans la seconde $(P<0,01)$. Cette augmentation du gain moyen quotidien résulte des différences significatives des gains moyens quotidiens au cours des 6 premières et 6 dernières semaines.

\section{Quantités ingérées}

Dans les deux expériences (tabl. 4, fig. 4) les animaux recevant le poloxalène ont ingéré davantage d'aliment (environ ro p. Ioo) que les taurillons des lots témoins. Cette différence est significative dans les deux cas, au seuil de 5 p. Ioo.

L'efficacité alimentaire a été identique pour les 2 lots dans la première expérience $(7,2 \mathrm{I}$ et $7, \mathrm{I} 7 \mathrm{~kg}$ d'aliment par $\mathrm{kg}$ de gain, respectivement pour les lots poloxalène èt témoin). Elle a été légèrement améliorée (différence non significative) pour le lot poloxalène dans la seconde $(8,36$ contre $8,8 \mathrm{I} \mathrm{kg})$.

En outre, aucune liaison entre les quantités ingérées et les fréquences d'apparition des météorisations n'a pu être mise en évidence.

\section{Poids, rendement et état d'engraissement des carcasses}

Les poids, les rendements en carcasse exprimés en pourcentage du poids vif vide ainsi que l'état d'engraissement estimé par la proportion de dépôts adipeux périrénaux, n'ont pas varié de façon significative entre les lots témoin et poloxalène (tabl. 5).

TABLEAU 5

Poids, rendement et état d'engraissement des carcasses

( $2^{\mathrm{e}}$ expérience)

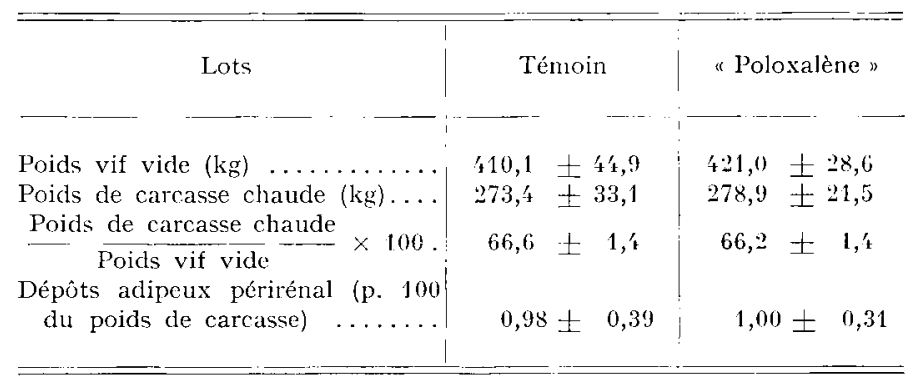

Toutefois, à poids de carcasses constants, les foies des animaux recevant le poloxalène étaient significativement plus lourds $(7,2$ I contre $6,20 \mathrm{~kg})$. 


\section{DISCUSSION}

Les observations réalisées dans les deux expériences ont donné des résultats concordants :

Elles ont permis de confirmer que l'utilisation d'une ration condensée durant une longue période entraîne des météorisations d'intensités élevées. D'autre part l'incorporation du poloxalène dans la ration (à raison de 0,1 ou 0,2 p. Ioo) diminue le nombre de cas de météorisations, particulièrement celles de forte intensité et en fin de période d'engraissement.

En outre, ces observations nous ont permis de mettre en évidence un fait nouveau: le poloxalène entraîne une augmentation significative des quantités d'aliments ingérées ( $\mathrm{l} \mathrm{kg}$ supplémentaire environ en moyenne par animal et par jour dans les deux expériences) et du gain de poids vif moyen quotidien. Toutefois, ces différences de gain de poids résultent essentiellement des différences de quantités ingérées, l'efficacité alimentaire n'ayant pratiquement pas été modifiée.

Il est vraisemblable que l'augmentation de la consommation résulte de la diminution de la fréquence des météorisations et surtout d'une diminution de l'accumulation des gaz dans le rumen. On sait, en effet, l'importance des phénomènes physiques de distension du rumen dans la régulation de l'appétit des ruminants (BALCH et Camplinc, ig62).

Qu'un effet apparemment aussi important n'ait jamais été signalé peut apparaître surprenant. Dans le travail de SHONE (1965) et dans celui de BARTLEY et MEYER (r968), seul le point de vue pathologique a été envisagé, tandis que GEISSLER et Tromas (Ig66) au cours de deux essais n'ont observé aucune influence du poloxalène sur la croissance de taurillons ( 880 et $800 \mathrm{~g} / \mathrm{jour}$ ) ; cependant, dans ces expériences, les aliments étaient distribués en quantité limitée et l'influence du poloxalène sur l'appétit ne pouvait donc se manifester.

Cet effet nouveau mériterait d'être confirmé par une étude plus approfondie, sur de plus grands effectifs et avec différents types de régimes.

Enfin, ces résultats permettent d'envisager une voie nouvelle actuellement peu explorée, de l'augmentation de l'appétit des ruminants.

Rę̧u pour publication en février 1971.

\section{REMERCIEMENTS}

Nous remercions MM. JARrige et Poutous pour les critiques et suggestions qu'ils ont formulées à la lecture de ce mémoire. 


\section{SUMMARY}

\section{THE INFLUENCE OF POLOXALENE ON BLOAT, THE AMOUNT OF FEED INGESTED AND THE GROWTH OF YOUNG BULLS RECEIVING CONDENSED RATIONS}

During two successive experiments, we studied the influence of poloxalene on bloat, the amount of feed ingested and the growth of $\mathrm{I} 23$ young bulls whose characteristics are given in table $I$ and which received condensed rations distributed ad libitum.

The incorporation of o.I p. Ioo and $0.2 \mathrm{p}$. I oo poloxalene in the diet has given the following results during the experimental period:

- a significant decrease in the number of cases of bloat and in particular in the number of serious cases whose frequency increases notably in the controls when the diet is continued for more than about 3 months (fig. I and 2 , table 3 ).

- a significant increase in the average quantity of feed ingested (fig. 4, table 4).

- a significant increase in the daily average weight gain (fig. 3 , table 4) linked with an increase in food intake.

The use of such substances that reduce the accumulation of gases in the rumen would give a new method for increasing the appetite of ruminants.

\section{RÉFÉRENCES BIBLIOGRAPHIQUES}

Balch C. C., CAmpling R. C., I962. Regulation of volontary food intake in ruminants. Nutr. Abstr. Rev., 32, 669-686.

Bartley E. E., I965a. Bloat in cattle. VI. Prevention of legume bloat with a nonionic surfactant. J. Dairy Sci., 48, 102-104.

Bartley F. E., I965 $b$. An analysis of the bloat complex and progress toward its prevention. J.Am. Vet. Med. Ass., 147, I 397 -I 402.

Bartley lE. E., Lippke MT, Pfost H. B., Nijweide R. J., Jacobsox N. L., Meyer R. M., ig65. Bloat in cattle. $X$. Efficacy of poloxalene in controlling alfalfa bloat in dairy steers and in lactating cows in commercial dairy herds. J. Dairy Sci., 48, I657-1662.

Bartley Ji. E., Meyer R. M., I968. Feedlot bloat prevention with poloxalene. J. anim. Sci., 26, 9 I3.

Bartley li. E., Stiles I). A., Meyer R. M., Scheidy S. F., Clark J. G., Boren F. W., I967. Poloxalene for treatment of cattle with alfalfa bloat. J. Am. Vet. Med. Ass., 151, 339-343.

Bartley E. E., YaDAVA I. S., I $96 \mathrm{r}$. Bloat in cattle. IV. The role of bovine saliva, plant mucilages and animal mucins. J. anim. Sci., 20, 648-653.

Blaxter K. L., 1962. The energy metabolism of ruminants. Hutchinson Ed. London, 329 pp.

Demarquilly C., Journet M., i967. Valeur alimentaire des foins conderisés. I. Influence de la nature du foin et de la finesse de broyage sur la digestibilité et la quantité ingérce. Ann. Zootech., 16, I23-I5o.

Ferrando R., 1968. Pathologie des ruminants exploités intensivement. World. Rev. Anim. Prod., 4, I6-29.

Frebling J., Poujardieu B., Vissac 13., Béranger C., Teissier J. H., ig67. Stations de sélection bovine. Compte rendu technique $\mathrm{n}^{\circ} \mathrm{I}$, note générale. Bull. Techn. Inf. Ingrs Serv. agric., 224, $887-894$.

Geissler B. R., Thomas O. O., 1966. Poloxalene as a bloat preventative for wintering calves on barley and alfalfa rations. J. anim. Sci., 25, 589 .

Gordon J. G., 1958. The relationship between fineness of grinding of food and rumination. J. A gric. Sci., 41, $78-8$ o.

Helmer L. G., Lippke H., Bartley E. E., Jacobson N. L., Meyer R. M., Pfost H. B., ig65. Mode of action of poloxalene in preventing alfalfa bloat and its effects on milk production, feed intake, health and rumen fermentation. J. Dairy Sci., 48, 800 .

Journet M., Demarquilly C., ig67 Valeur alimentaire des foins condensés. II. Influence du broyage et de la mise en agglomérés sur la digestion du foin de luzerne dans le rumen. Ann. Zootech., 16, 307-32I.

Kick C. H., Gerlaugh P., 1936. Proc. Amer. Soc. Anim. Prod,, 28th annual meeting, p. 93. Cité par GORDON, 
Larvor P., I967. Accidents entraînés par l'alimentation à base de fourrages agglomérés ou de céréales. Journées d'Information de l'Institut Technique des Céréales et Fourrages. Paris $24^{-26}$ janvier, 277-288.

Malterre C., Vérité R., 1969. (Résultats non publiés).

Mendel V. E., Boda J.-M., I96r. Physiological studies of the rumen with emphasis on the animal factors associated with bloat. J. Dairy Sci., 44, $188 \mathrm{I}-1898$.

Meyer R. M., Bartley E. E., Morrill J. L., Stewart W. E., I964. Salivation in cattle. I. Food and animal factors affecting salivation and its relation to bloat. J. Dairy Sci., 47, I339-I345.

Meyer R. M., Helmer L. G., Bartley E. E., ig65. Bloat in cattle. VIII. Extent of elimination in milk and body tissues of ${ }^{14}$ Clabeled poloxalene used to prevent legume bloat. J. Dairy Sci., 48, 503-505.

Putnam P. A., Yarns D. A., Davis R. E., I966. Effect of pelleting rations and hay : grain ratio on salivary secretion and ruminal characteristics of steers. J. Anim. Sci., 25, i I $76-1180$.

RUCKEbUSCH Y., I967. Étude polygraphique des variations spontanées ou provoquées du comportement alimentaire chez les petits ruminants. Arch. Sci. Physiol., 21, 449-473.

Shone D. K., I965. Poloxalene as prophylactic and therapeutic agent for bloat in cattle fed on high concentrate diets. J. S. Afr. Vet. Méd. Ass., 36, 373-375.

Van Horn H. H., Bartley E. E., r96r. Bloat in cattle. I. Effect of bovine saliva and plant mucin on frothing rumen contents in alfalfa bloat. J. anim., Sci., 20, 85-87. 